Bundesgesundheitsbl $2017 \cdot 60: 286-291$ DOI 10.1007/s00103-016-2501-x Online publiziert: 4. Januar 2017 (c) Der/die Autor(en) 2017. Dieser Artikel ist eine Open-Access-Publikation.

CrossMark

Kerstin Stephan

Bundesinstitut für Arzneimittel und Medizinprodukte (BfArM), Bonn, Deutschland

\title{
Pharmakologische Wirkung als Abgrenzungskriterium
}

Die Verbraucher werden nicht zuletzt auch durch die älter werdende Gesellschaft gesundheitsbewusster und interessieren sich immer mehr für eine gesundheitserhaltende, gesundheitsfördernde und auch krankheitsrisikominimierende Ernährung. Dabei spielen Nahrungsergänzungsmittel eine immer größere Bedeutung. Das Angebot ist vielfältig und teilweise sehr komplex. Nicht selten werden auch krankheitslindernde oder auch -heilende Wirkversprechen assoziiert. Dies führt dazu, dass auch neben der Produktauslobung die tatsächliche Wirkungsweise von Arzneimitteln und Nahrungsergänzungsmitteln häufig kontrovers diskutiert wird oder oft Gegenstand von gerichtlichen Auseinandersetzungen ist. Eine korrekte Produkteinstufung stellt daher Behörden, Hersteller, aber auch Fachkreise und Verbraucher vor eine große Herausforderung. Denn nicht zuletzt das Marktzugangsverfahren und die Produktüberwachung von Arzneimitteln und Nahrungsergänzungsmitteln unterliegen sehr unterschiedlichen Verfahren und gesetzlichen Vorgaben.

\section{Gesetzliche Definitionen}

2002 wurde mit der Richtlinie 2002/46/EG [1] eine gesetzliche Definition für Nahrungsergänzungsmittel geschaffen, die mit der Nahrungsergänzungsmittelverordnung in deutsches Recht übernommen wurde:

Der Ausdruck „Nahrungsergänzungsmittel" bezeichnet Lebensmittel, die dazu bestimmt sind, die normale Ernährung $z u$ ergänzen, und die aus Einfach-oder Mehrfachkonzentraten von Nährstoffen oder sonstigen Stoffen mit ernährungsspezifischer oder physiologischer Wirkung bestehen und in dosierter Form in den Verkehr gebracht werden, d.h. in Form von z.B. Kapseln, Pastillen, Tabletten, Pillen und anderen ähnlichen Darreichungsformen, Pulverbeuteln, Flüssigampullen, Flaschen mit Tropfeinsätzen und ähnlichen Darreichungsformen von Flüssigkeiten und Pulvern zur Aufnahme in abgemessenen kleinen Mengen; ...

Somit werden neben Nährstoffen wie Vitaminen und Mineralstoffen auch sonstige Stoffe mit ernährungsspezifischer oder physiologischer Wirkung, wie beispielsweise Aminosäuren, Fettsäuren oder Ballaststoffe, als Bestandteil von Nahrungsergänzungsmitteln, die die Zweckbestimmung der Ergänzung der allgemeinen Ernährung haben, aufgeführt.

Dagegen sind Arzneimittel nach dem Arzneimittelgesetz [2] wie folgt definiert:

Arzneimittel sind Stoffe oder Zubereitungen aus Stoffen,

1. die zur Anwendung im oder am menschlichen oder tierischen Körper bestimmt sind und als Mittel mit Eigenschaften zur Heilung oder Linderung oder zur Verhütung menschlicher oder tierischer Krankheiten oder krankhafter Beschwerden bestimmt sind oder

2. die im oder am menschlichen oder tierischen Körper angewendet oder einem Menschen oder einem Tier verabreicht werden können, um entweder

a) die physiologischen Funktionen durch eine pharmakologische, immunologische oder metabolische Wirkung wiederherzustellen, zu korrigieren oder $z u$ beeinflussen oder

b) eine medizinische Diagnose zu erstellen.
Die Definition für Arzneimittel gemäß Artikel 1 Nr. 2 der Richtlinie 2001/83/EG [3] ist fast inhaltsgleich in das deutsche Gesetz übernommen worden. Die Begriffe der Linderung und der krankhaften Beschwerde wurden im Arzneimittelgesetz ergänzt. Folglich sind auch palliativ wirkende Produkte den Arzneimitteln zuzuordnen.

Somit unterscheiden sich Nahrungsergänzungsmittel von Arzneimitteln zum einen durch das für ein konkretes Produkt bestimmte Anwendungsgebiet, nämlich die Nahrungsergänzung des gesunden Verbrauchers für Nahrungsergänzungsmittel gegenüber der Heilung, Linderung oder Verhütung von Krankheiten als Zweckbestimmung von Arzneimitteln (Präsentationsarzneimittel), zum anderen durch die Wirkungsweise, die das Produkt am oder im menschlichen Körper ausübt, und zwar die ernährungsspezifische oder physiologische Wirkung für Nahrungsergänzungsmittel und die pharmakologische Wirkung, die typisch für Arzneimittel ist (Funktionsarzneimittel).

\section{Deutungsmöglichkeiten unbestimmter Rechtsbegriffe}

Da für viele Nahrungsergänzungsmittel häufig keine nähere Zweckbestimmung angegeben ist, fällt der Schwerpunkt der Abgrenzungsarbeit oft auf die Prüfung der Wirkungsweise.

Liegt nachweislich eine pharmakologische, metabolische oder immunologische Wirkung des konkreten Produktes vor, so ist dies als Arzneimittel einzuordnen. Ist diese Wirkungsweise nicht nachweisbar, handelt es sich ggf. um ein Nahrungsergänzungsmittel. Wenn für ein Produkt eine medizinische Zweck- 
bestimmung vorliegt, so kann es sich jedoch auch um ein Medizinprodukt handeln, wenn die bestimmungsgemäße Hauptwirkung im Unterschied zu Arzneimitteln nicht auf pharmakologischem (resp. metabolischem oder immunologischem) Weg erzielt wird. So sind Präparate mit Simethicon zur Behandlung von Magen-Darm-Beschwerden aufgrund der Beeinflussung der Oberflächenspannung des Nahrungsbreis, die als physikochemische Wirkung zu bezeichnen ist, als Medizinprodukte einzustufen.

Es ist festzustellen, dass weder die Begriffe „ernährungsspezifische“ oder "physiologische Wirkung“ noch der Begriff „pharmakologische (resp. metabolische oder immunologische) Wirkung" gesetzlich definiert sind. So finden sich weder in den deutschen Gesetzen (Arzneimittelgesetz, Nahrungsergänzungsmittelverordnung) noch in den europäischen Verordnungen oder Richtlinien (VO (EU) 178/2002 [4], Richtlinie 2002/46, Richtlinie 2001/83) entsprechende Definitionen oder Erläuterungen.

Folglich führen diese unbestimmten Rechtsbegriffe in der praktischen Anwendung zu erheblichen Problemen.

Die „Medical Devices Expert Group on Borderline and Classification", eine Expertengruppe der Europäischen Kommission für die Beurteilung von Medizinprodukten, hat in der Leitlinie „MEDDEV 2.1/3 rev. 3“ [5] folgende Definition für den Begriff "pharmakologisch“ im Hinblick auf die Abgrenzung von Arzneimitteln zu Medizinprodukten formuliert:

"Pharmacological means" is understood as an interaction between the molecules of the substance in question and a cellular constituent, usually referred to as a receptor, which either results in a direct response, or which blocks the response to another agent. Although not a completely reliable riterion, the presence of a dose-response correlation is indicative of a pharmacological effect.

Diese Definition wird auch häufig bei der Abgrenzung von Arzneimitteln zu Lebensmitteln resp. Nahrungsergänzungsmitteln herangezogen, auch wenn sie diesbezüglich nicht immer hilfreich erscheint.

Laut Pschyrembel [6] - klinisches Wörterbuch - wird unter einer pharmakologischen Wirkung die Fähigkeit von Stoffen, in Wechselwirkung mit dem menschlichen Organismus treten zu können, definiert.

In Lehrbüchern zur Pharmakologie und Toxikologie $[7,8]$ wird die Pharmakologie als die Wissenschaft von den Wechselwirkungen zwischen Stoffen und Lebewesen bezeichnet. Auch diese Definitionen erscheinen für die Abgrenzung von Arzneimitteln zu Lebensmitteln resp. Nahrungsergänzungsmitteln wenig nützlich.

Denn auch Lebensmittelinhaltsstoffe, wie beispielsweise Koffein, haben eine diesen Definitionen entsprechende pharmakologische Wirkung.

Koffein interagiert mit Adenosinrezeptoren. Adenosin drosselt im Gehirn die Freisetzung von bestimmten Neurotransmittern wie Glutamat und Dopamin, die in den Synapsen für die Weiterleitung eines Nervenreizes zuständig sind. Da Koffein einen Großteil der Adenosinrezeptoren besetzt, wird diese Hemmung teilweise aufgehoben. Es kommt zur vermehrten Ausschüttung der Transmitter, wodurch die Erregungsweiterleitung von Nervenimpulsen erleichtert wird. So wirkt das Genussmittel zentral erregend.

Auch Alkohol weist nachweislich eine pharmakologische Wirkung auf. So werden Rezeptoren und Signalwege von $\gamma$-Aminobuttersäure (GABA), Acetylcholin und Glutamat beeinflusst. Alkohol aktiviert den neuronalen Nikotinrezeptor. Es kommt zu zentralnervösen Wirkungen.

Dennoch wird Alkohol in Form von Wein, Bier oder Spirituosen zweifelsfrei als Lebensmittel angesehen.

\section{Begriffsauslegung der Rechtsprechung}

\section{Pharmakologische oder} therapeutische Wirkung als Kriterium

Die pharmakologischen Eigenschaften eines Stoffes im Sinne seiner Wirkmög- lichkeiten sind seit 1983 in der Rechtsprechung des Europäischen Gerichtshofs (EuGH) für die Einstufung eines Produktes als Arzneimittel das wichtigste Kriterium [9]. Dabei muss die Wirkung auf den Stoffwechsel nennenswert sein und die Funktionsbedingungen des menschlichen Körpers müssen wirklich beeinflusst werden [10].

Es ist häufig versucht worden, den Begriff der pharmakologischen Wirkung im Hinblick auf die Abgrenzung von Arzneimitteln zu anderen Produktgruppen, wie z. B. Nahrungsergänzungsmitteln, näher und insbesondere eindeutiger $\mathrm{zu}$ beschreiben.

So hat der EuGH mit Urteil vom 9.6.2005 [11] im Zusammenhang mit einem Vorabentscheidungsersuchen des Oberverwaltungsgerichts für das Land Nordrhein-Westfalen zur Einstufung verschiedener Produkte u. a. ein Präparat mit Milchsäurebakterien (Lactobact omni FOS) und ein Präparat mit Polyphenolen (OPC) und zur Frage der Definition des Begriffes ,pharmakologische Wirkung“ festgestellt, dass

die pharmakologischen Eigenschaften eines Erzeugnisses der Faktor sind, auf dessen Grundlagen die mitgliedstaatlichen Behörden ausgehend von der Wirkungsmöglichkeit des Erzeugnisses zu beurteilen haben, ob es im Sinne des Artikels 1 Nummer 2 Absatz 2 der Richtlinie 2001/83 dazu bestimmt ist, im oder am menschlichen Körper zur Erstellung einer ärztlichen Diagnose oder zur Wiederherstellung, Besserung oder Beeinflussung der menschlichen physiologischen Funktionen angewandt $z u$ werden.

Somit ist die Prüfung der pharmakologischen Wirkung eines Präparates bei der Beurteilung als Arzneimittel nach Funktion ausschlaggebend.

Grundsätzlich liegt die Einstufung konkreter Präparate in der Zuständigkeit der Mitgliedstaaten. Daher haben in dem o. g. Verfahren nicht der EuGH, sondern die nationalen Gerichte entschieden.

In dem entsprechenden Rechtsverfahren zu den o.g. Produkten hat das Oberverwaltungsgericht NordrheinWestfalen (OVG NRW) [12] festgestellt, dass der Begriff der pharmakologischen 
Wirkung ein untaugliches Kriterium zur Abgrenzung von Lebensmitteln und Arzneimitteln sei und in diesen Fällen offensichtlich nicht weiterführe. Aus Sicht des Gerichts sind die Begriffe der "pharmakologischen, immunologischen und metabolischen Wirkung “im Hinblick auf die Unterscheidung von Arzneimitteln $\mathrm{zu}$ Medizinprodukten geschaffen worden und bei dieser Abgrenzung durchaus brauchbar. Denn die von einem Molekül eines Stoffes ausgelöste Zellreaktion (siehe Definition der Leitlinien MEDDEV 2.1/3 rev. 3) ist als Kriterium gegenüber der rein physikalischen Wirkung eines Medizinproduktes durchaus heranzuziehen. Das Auslösen einer Zellreaktion durch Moleküle eines Stoffes ist bei der Unterscheidung eines Arzneimittels $\mathrm{zu}$ einem Lebensmittel kein taugliches Kriterium, weil Letztere in aller Regel anders als Medizinprodukte keine rein physikalische Wirkung haben, sondern - ebenso wie Arzneimittel - die zuvor beschriebene Reaktion auslösen können, indem sie nach Aufnahme vom Körper im Wege des Stoffwechsels umgesetzt werden. Das Oberverwaltungsgericht (OVG) zielt daher für die Einordnung als Funktionsarzneimittel entscheidend darauf $a b$, ob das jeweilige Produkt geeignet ist, einen therapeutischen Zweck $\mathrm{zu}$ erfüllen. Entsprechend wurden die streitgegenständlichen Erzeugnisse vom Oberverwaltungsgericht als Lebensmittel eingestuft.

Das Bundesverwaltungsgericht (BVerwG) [13] hält in seinem Urteil zu den gleichen Produkten jedoch dieses Vorgehen für rechtssystematisch bedenklich und verweist darauf, dass der EuGH wiederholt bei Abgrenzungen von Arzneimitteln $\mathrm{zu}$ anderen Produkten, wie Vitaminpräparaten, die in der rechtlichen Definition des Arzneimittels vorkommenden Begriffe der pharmakologischen, immunologischen und metabolischen Wirkung herangezogen hat.

Die Änderungsrichtlinie 2004/27/EG hat die pharmakologische (und auch die immunologische und metabolische) Wirkung ausdrücklich in die Definition einbezogen.

So hält das BVerwG es für richtig, dass ein Erzeugnis, das geeignet ist, therapeutische Zwecke zu erfüllen, in jedem

Bundesgesundheitsbl 2017 -60:286-291 DOI 10.1007/s00103-016-2501-x

๑ Der/die Autor(en) 2017. Dieser Artikel ist eine Open-Access-Publikation.

\section{K. Stephan}

\section{Pharmakologische Wirkung als Abgrenzungskriterium}

\section{Zusammenfassung}

Durch den immer größer werdenden Gesundheitsmarkt und das größer werdende Gesundheitsbewusstsein der Bevölkerung werden immer mehr Nahrungsergänzungsmittel neben Arzneimitteln auf den Markt gebracht. Dabei konzentriert sich die Abgrenzung von Arzneimitteln zu Nahrungsergänzungsmitteln neben der ausgelobten Zweckbestimmung auf die Wirkung des jeweiligen Präparates. Ein Arzneimittel weist laut gesetzlicher Definition eine pharmakologische Wirkung auf, ohne dass diese näher definiert wird. Daher haben sich bereits viele Gerichte mit der Auslegung des Begriffs der pharmakologischen Wirkung, der als Oberbegriff auch die metabolische oder immunologische Wirkung beinhaltet, beschäftigt und versucht diesen genauer zu beschreiben.

Festzuhalten ist, dass die pharmakologische Wirkung, d. h. eine Wechselwirkung des Inhaltsstoffes eines Arzneimittels mit zellulä- ren Strukturen des menschlichen Körpers, anders als die Wirkung eines Lebensmittels - die physiologischen Funktionen erheblich beeinflussen muss, also eine weitergehende und zielgerichtetere Wirkung auf den menschlichen Körper und seine Funktionen ausüben muss, als das mit dem Verzehr einer üblichen Menge eines Lebensmittels möglich wäre. Auch muss diese Beeinflussung der physiologischen Funktionen des Körpers positiv gerichtet, also der menschlichen Gesundheit zuträglich sein. Produkte, die nur aus Rauschzwecken konsumiert werden und sogar eine gesundheitsschädliche Wirkung haben, werden trotz ihres pharmakologischen Wirkmechanismus nicht als Arzneimittel eingestuft.

\section{Schlüsselwörter}

Pharmakologische Wirkung · Funktionsarzneimittel · Nahrungsergänzungsmittel · Abgrenzung

\section{Pharmacological properties as criterion of demarcation}

\section{Abstract}

Because of the growing healthcare market and the increasing health consciousness of the population, more and more food supplements are being placed on the market in addition to medicinal products. Concerning the demarcation of medicinal products to food supplements, not only is the intended purpose of the product critical, but the mode of action of the respective preparation also plays an important role. Following the legal definition, a drug has a pharmacological effect, without it being further defined. Therefore, many courts have already tried to interpret the term pharmacological effect, including the terms metabolic or immunologic effect, and try to describe this in more detail.

It should be noted that the pharmacological effect, which means an interaction of the substance of a medicinal product with cellular constituent of the human body - unlike the effect of a foodstuff - must significantly affect the physiological functions and must therefore exert targeted effects on the human body and its functions, more than with the consumption of a conventional quantity of a food would be possible. These modifying physiological functions of the body must be addressed positively, which means beneficial to human health. Products which are consumed only for euphoria purposes, or even have a harmful effect, shall not be classified as medicinal products, despite their pharmacological mechanism of action.

\section{Keywords}

Pharmacological properties - Medicinal product by function - Food supplement . Demarcation
Fall ein Arzneimittel ist. Fehlt jedoch diese Eignung, so ist nicht ausgeschlossen, dass es sich dennoch um ein Funktionsarzneimittel handelt [14]. Somit ist auch dann ein Produkt als Funktionsarzneimittel einzustufen, wenn zwar keine klinisch relevante therapeutische Wirk- samkeit, jedoch eine pharmakologische Wirkung nachweislich vorliegt. In diesem Fall würde es sich möglicherweise um ein unwirksames Arzneimittel handeln. 
Das BVerwG versucht in diesen Urteilen den Begriff der pharmakologischen Wirkung näher zu beschreiben:

Eine pharmakologische Wirkung ist eine gezielte Steuerung von Körperfunktionen von außen, sie ist nicht mit der unspezifischen Aufnahme von Nährstoffen über natürliche Nahrungsmittel vergleichbar, bei der der Körper die benötigten Bestandteile selbst identifiziere und modifiziere.

Auch müssen die erhebliche Beeinflussung der Funktionsbedingungen des menschlichen Körpers und das Vorliegen erheblicher pharmakologischer Wirkungen durch belastbare wissenschaftliche Erkenntnisse belegt sein.

Zwar ist kein positiver Wirksamkeitsnachweis erforderlich, wie er Voraussetzung einer Arzneimittelzulassung wäre, es muss aber zumindest ein halbwegs gesicherter wissenschaftlicher Erkenntnisstand vorliegen, der einen tragfähigen Rückschluss auf die Wirkungen des jeweiligen Produktes erlaubt [15].

Diese Formulierung lässt die Frage aufkommen, was denn ein „halbwegs gesicherter wissenschaftlicher Erkenntnisstand“ ist. Welche Art von Evidenzgrad müssen die vorgelegten wissenschaftlichen Daten für den Beleg der pharmakologischen Wirkung aufweisen?

Das Gericht betont, dass eine Einstufung eines Präparates nicht „auf Verdacht" erfolgen darf. So hat auch der Generalanwalt in seinen Schlussanträgen zu der vom OVG NRW eingeholten Vorabentscheidung [16] ausdrücklich vor einer zu extensiven Auslegung und Anwendung der Definition des Arzneimittels gewarnt. Er hat gefordert, es müsse eine ausreichende Sicherheit dafür bestehen, dass Produkte, die angeblich eine Wirkung als Arzneimittel haben, diese Wirkung auch tatsächlich aufweisen.

\section{Aufbereitungsmonografien des} ehem. Bundesgesundheitsamts als Beleg

Das BVerwG sieht die Aufbereitungsmonografien des Bundesgesundheitsamts als belastbare Grundlage zum Beleg der pharmakologischen Wirkung an [17]. Diese sind auf einer gesetzlichen Grundlage von einem kompetenten Ex- pertengremium erstellt und vom damaligen Bundesgesundheitsamt anerkannt und veröffentlicht worden. $\mathrm{Ob}$ die dort getroffenen Aussagen inzwischen überholt sein könnten, ist entsprechend $\mathrm{zu}$ belegen.

Liegen keine Aufbereitungsmonografien vor, sind entsprechende Daten vorzulegen. Ob diese dann einen tragfähigen Rückschluss auf die Wirkungen des jeweiligen Präparates erlauben, ist im Einzelfall zu prüfen und könnte in einigen Fällen strittig sein.

\section{Erheblichkeitsschwelle}

Mit dem Urteil vom 16.5.2007 [18] hat das BVerwG den Begriff der „Erheblichkeitsschwelle" verwendet. In diesem Urteil, bei dem es um die Einstufung eines Kosmetikums ging, hat das Gericht festgestellt, dass

pharmakologische oder metabolische Wirkungen eines Stoffes nur dann dessen $\mathrm{Zu}$ ordnung zu den Arzneimitteln rechtfertigen, wenn sie die „Erheblichkeitsschwelle“ überschreiten. Eingriffe in die Körperfunktionen, die völlig unerheblich sind, können dagegen die Zuordnung zu den Arzneimitteln nicht rechtfertigen.

Wird somit ein potenziell pharmakologischer Stoff unterhalb der belegten pharmakologischen Wirkung dosiert, kann es sich nicht um ein Funktionsarzneimittel handeln.

Ähnlich argumentierte auch der Europäische Gerichtshof mit Urteil vom 15.11.2007 [19]. So dürfen nur dann Stoffe als Arzneimittel nach Funktion eingestuft werden, wenn sie sich nennenswert auf den Körper auswirken. Gehen die Auswirkungen auf die physiologischen Funktionen nicht über die Wirkungen hinaus, die ein in angemessener Menge verzehrtes Lebensmittel auf diese Funktion haben kann, handelt es sich nicht um eine nennenswerte Beeinflussung und somit ist das fragliche Produkt nicht als Funktionsarzneimittel einzustufen.

Auch in seinem Urteil vom 15.01.2009 [20] stellt der Europäische Gerichtshof klar, dass ein Produkt nicht als Arzneimittel eingestuft werden kann, wenn es aufgrund seiner Zusammensetzung - einschließlich der Dosierung seiner
Wirkstoffe - und bei bestimmungsgemäßer Anwendung die physiologischen Funktionen nicht in nennenswerter Weise durch eine pharmakologische, immunologische oder metabolische Wirkung wiederherstellt, korrigiert oder beeinflusst. Beispielhaft wäre hier ein Präparat mit Alpha-Liponsäure (Dosierung $300 \mathrm{mg} / \mathrm{Tag}$ ) zu nennen, das als Nahrungsergänzungsmittel in den Verkehr gebracht wird, das aber aufgrund der belegbaren pharmakologischen Wirkung als Arzneimittel eingestuft wird. Auch ist hier die sog. Erheblichkeitsschwelle überschritten, denn um eine Menge von $300 \mathrm{mg}$ Alpha-Liponsäure aufzunehmen, müsste man $1,5 \mathrm{~kg}$ Molkepulver, 2,4 kg frischen Spinat oder $3 \mathrm{~kg}$ schlachtfrische Leber verzehren. Dies ist als nicht angemessene Verzehrmenge anzusehen [21].

\section{Toxische Wirkung als Kriterium}

Mit der Frage, ob auch eine negative Wirkung, also eine toxische Wirkung, als pharmakologische Wirkung anzusehen ist, hat sich zunächst das Oberverwaltungsgericht des Saarlandes beschäftigt [22] und kommt zu dem Ergebnis, dass es sich bei einer toxischen Wirkung, die eine Gesundheitsgefahr darstellen kann, um eine negativ pharmakologische Wirkung handelt und es sich dann um ein Funktionsarzneimittel handelt. Die besonderen Gesundheitsgefahren (hier die Förderung von Entzündungsprozessen durch ein niedrig dosiertes Weihrauchpräparat) gerade von Arzneimitteln erfordern im Zweifel die Anwendung des strengeren Gesundheitsrechts gegenüber dem weniger strengen Lebensmittelrecht.

Die Vorlagefrage des Bundesverwaltungsgerichts:

ist der Begriff des Arzneimittels in Art. 1 Nr. 2 der Richtlinie 2001/83 dahin auszulegen, dass ein zum menschlichen Verzehr bestimmtes und als Nahrungsergänzungsmittel bezeichnetes Produkt ein Funktionsarzneimittel ist, wenn es Stoffe enthält, die bei Beachtung der auf der Verpackung aufgedruckten Verzehrempfehlung in der im Produkt enthaltenen niedrigen Dosierung gesundheitsgefährdend sind, ohne therapeutische Wirkungen erzielen 
zu können, die aber in hoher Dosierung therapeutisch wirksam sind[,]

wurde dann vom Europäischen Gerichtshof mit Urteil vom 30.4.2009 [23] beantwortet, dass

Art. 1. Nr. 2 der Richtlinie 2001/83/EG [...] dahin auszulegen ist, dass ein Erzeugnis, das einen Stoff enthält, der in einer bestimmten Dosierung eine physiologische Wirkung hat, kein Funktionsarzneimittel ist, wenn es in Anbetracht seiner Wirkstoffdosierung bei normalem Gebrauch gesundheitsgefährdend ist, ohne jedoch die menschlichen physiologischen Funktionen wiederherstellen, korrigieren oder beeinflussen zu können.

Folglich wurde das Weihrauchpräparat nicht als Funktionsarzneimittel eingestuft.

Dass die pharmakologische Wirkung im Sinne der Arzneimitteldefinition als eine positiv gerichtete Wirkung zu verstehen ist und nicht jedwede pharmakologische Wirkung zur Einstufung als Funktionsarzneimittel ausreicht, wird auch in einem späteren Urteil des Europäischen Gerichtshofs zu sog. Legal Highs [24] deutlich.

Hierbei wurde dem EuGH die Frage vorgelegt, ob der Begriff des Funktionsarzneimittels nach der Richtlinie 2001/83 auch auf Stoffe zutrifft, deren Wirkungen sich darauf beschränken, die physiologischen Funktionen lediglich zu beeinflussen, ohne positiv auf sie einzuwirken. Diese Stoffe werden zu Rauschzwecken konsumiert und sind dabei gesundheitsschädlich.

Das Gericht hat diese Frage verneint:

Art. 1. Nr. 2 Buchst. $b$ der Richtlinie 2001/83 [...] ist dahin auszulegen, dass davon Stoffe [...] nicht erfasst werden, deren Wirkungen sich auf eine schlichte Beeinflussung der physiologischen Funktionen beschränken, ohne dass sie geeignet wären, der menschlichen Gesundheit unmittelbar oder mittelbar zuträglich zu sein, die nur konsumiert werden, um einen Rauschzustand hervorzurufen, und die dabei gesundheitsschädlich sind[.]

Auch das Bundesverwaltungsgericht hat bei der sog. elektrischen Zigarette ähnlich entschieden [25]: Danach sind Nikotin-
Liquids, wie sie in sog. elektrischen Zigaretten verwendet werden, nicht als Funktionsarzneimittel anzusehen. Zwar kann Nikotin eine nennenswerte pharmakologische Wirkung auf den Stoffwechsel entfalten. Bei Gesamtschau aller Produktmerkmale ist das Produkt jedoch eher als Genussmittel einzustufen. Ein therapeutischer Nutzen des Nikotins in der elektrischen Zigarette hat das Gericht als wissenschaftlich nicht belegt angesehen.

Nach diesen beiden Urteilen kann man den Eindruck gewinnen, dass der Begriff der pharmakologischen Wirkung eines Produktes in Bezug auf die Einstufung als Arzneimittel nicht völlig losgelöst von der medizinischen Zweckbestimmung bewertet werden darf. $\mathrm{Zu}$ mindest eine therapeutische Eignung muss angenommen werden können oder eine für die Gesundheit förderliche Beeinflussung der physiologischen Funktionen liegt vor.

Ob dies im Widerspruch zur Zweiteilung des Arzneimittelbegriffs in der gesetzlichen Definition steht, ist zu prüfen.

Auch bleibt in diesem Zusammenhang die Frage, wie mit Lifestyleprodukten umzugehen ist. Diese haben meistens einen gut nachgewiesenen pharmakologischen Wirkungsmechanismus; der therapeutische Nutzen im Sinne einer Krankheitsbehandlung ist aber durchaus fraglich.

Als Beispiel hierfür wären Mittel zur Förderung des Haarwachstums ohne Diagnose einer Alopezie oder zur Reduzierung von Körpergewicht ohne Vorliegen einer Adipositas oder die Anwendung von Mitteln zur Glättung von Gesichtsfalten wie Botulinumtoxin aus rein kosmetischen Gründen zu nennen.

\section{Fazit}

Die Auslegung des Begriffs der pharmakologischen Wirkung im Sinne der Einstufung eines Produktes als Funktionsarzneimittel ist vielschichtig und immer im Einzelfall auch in Bezug auf die Abgrenzung zur anderen Produktkategorien zu bewerten. So sollte der Wirkungsmechanismus bei der Abgrenzung $\mathrm{zu}$ Medizinprodukten genau betrachtet werden. Das Wirkungsausmaß ist hierbei meistens nicht wesentlich.

Dagegen ist die pharmakologische Wirkung eines Erzeugnisses bei der Abgrenzung von Arzneimitteln zu Lebensmitteln resp. Nahrungsergänzungsmitteln nicht alleine durch den Wirkungsmechanismus zu klären. Hierbei spielt das Ausmaß, nämlich die „Erheblichkeit“, eine entscheidende Rolle. Ist die Wirkung zwar klar beschrieben, unterscheidet sich aber nicht von dem Verzehr einer üblichen Menge eines Lebensmittels, ist das Erzeugnis nicht als Arzneimittel einzustufen. Auch muss die Wirkung positiv gerichtet sein, d.h., sie muss der Gesundheit zuträglich sein und somit die physiologischen Wirkungen positiv beeinflussen.

Insgesamt weisen die Gerichte immer wieder darauf hin, dass die Einordnung eines Präparates als Arzneimittel gerechtfertigt sein muss und der Funktionsarzneimittelbegriff nicht $\mathrm{zu}$ weit auszulegen ist. In jedem Fall ist der Beleg der erheblichen pharmakologischen Wirkung durch belastbare wissenschaftliche Daten zu erbringen. Liegen diese nicht vor, ist eine Einstufung als Funktionsarzneimittel nicht möglich. Dies bedeutet aber in der Praxis ein Verwischen der Grenzen zwischen Arzneimittel und Nahrungsergänzungsmittel, sodass insbesondere der Verbraucher kaum einen Unterschied ausmachen kann.

Diese Vermischung der Zuordnungen ist jedoch zu hinterfragen, da Nahrungsergänzungsmittel zu den Lebensmitteln gehören und anders als Arzneimittel u. a. keiner behördlichen Zulassung vor Marktzugang und auch keiner engmaschigen Überwachung von möglichen Risiken nach Anwendung wie bei den Arzneimitteln („Pharmakovigilanz“) unterliegen. Dem Verbraucherschutz sollte hier mehr Beachtung gegeben werden.

Dieser Vermischung entgegenzuwirken und damit mehr Rechtssicherheit bei der Abgrenzung von Arzneimitteln zu Nahrungsergänzungsmitteln zu schaffen, könnte durch klare rechtsverbindliche Definitionen sowie Positivlisten für die „sonstigen Stoffe“ sowie Höchstmengen für Vitamine und Mineralstoffe, wie bereits in den Erwägungsgründen 11 bzw. 13 der Nahrungsergänzungsmittelricht- 
linie 2002/46/EG vorgeschlagen, erreicht werden. Hier ist der europäische, aber auch der nationale Gesetzgeber gefordert.

\section{Korrespondenzadresse}

\section{Dr. rer. nat. K. Stephan}

Bundesinstitut für Arzneimittel und

Medizinprodukte (BfArM)

Kurt-Georg-Kiesinger-Allee 3, 53175 Bonn,

Deutschland

kerstin.stephan@bfarm.de

\section{Einhaltung ethischer Richtlinien}

Interessenkonflikt. K. Stephan gibt an, dass kein Interessenkonflikt besteht.

Dieser Beitrag beinhaltet keine Studien an Menschen oder Tieren.

Open Access. Dieser Artikel wird unter der Creative Commons Namensnennung 4.0 International Lizenz (http://creativecommons.org/licenses/by/4.0/deed. de) veröffentlicht, welche die Nutzung, Vervielfältigung, Bearbeitung, Verbreitung und Wiedergabe in jeglichem Medium und Format erlaubt, sofern Sie den/die ursprünglichen Autor(en) und die Quelle ordnungsgemäß nennen, einen Link zur Creative Commons Lizenz beifügen und angeben, ob Änderungen vorgenommen wurden.

\section{Literatur}

1. Richtlinie 2002/46/EG des Europäischen Parlaments und des Rates vom 10. Juni 2002 zur Angleichung der Rechtsvorschrift der Mitgliedstaaten über Nahrungsergänzungsmittel. Amtsblatt Europ. Gemeinschaften L 183/51-57 vom 12. Juli 2002

2. Arzneimittelgesetz in der Fassung der Bekanntmachung vom 12. Dezember 2005 (BGBI. I S. 3394), das zuletzt durch Artikel 4 Absatz 11 des Gesetzes vom 18. Juli 2016 (BGBI.IS. 1666) geändert worden ist

3. Richtlinie 2001/83/EG des Europäischen Parlaments und des Rates vom 6. November 2001 zur Schaffung eines Gemeinschaftskodex für Humanarzneimittel. Amtsblatt Europ. Gemeinschaften L 311 vom 28.11.2011, S67

4. Verordnung (EG) Nr. 178/2002 des Europäischen Parlaments und des Rates vom28. Januar 2002 zur Feststellung der allgemeinen Grundsätze und Anforderungen des Lebensmittelrechts, zur Errichtung der Europäischen Behörde für Lebensmittelsicherheit und zur Feststellung von Verfahren zur Lebensmittelsicherheit. Amtsblatt Europ. GemeinschaftenL31/1-24 vom 1. Feb. 2002

5. MEDICAL DEVICES: Guidance document - Scope, field of application, definition - Borderline products, drug-delivery products and medical devices incorporating, as integral part, an ancillary medicinal substance or an ancillary human blood derivative: Guidelines relating to the application of the Council Directive 90/385/EEC on active implantable medical devices, the Council Directive 93/42/EEC on medical devices, MEDDEV 2.1/3 rev.3

6. Pschyrembel online (2016) Walter de Gruyter GmbH. https://www.pschyrembel.de. Zugegriffen: 16.09.2016

7. Aktories K, Förstermann U, Hofmann F, Starke K (2013) Allgemeine und spezielle Pharmakologie, S2

8. Mutschler E (2012) Arzneimittelwirkungen: Pharmakologie, klinische Pharmakologie, Toxikologie, S1

9. Urteile des Europäischen Gerichtshofes vom 30. November 1983 - Rs. 227/82, van Bennekom und vom 16. April 1991 Rs.- C-112/98, Upjohn

10. Urteil des Europäischen Gerichtshofes vom 16 April 1991 RS 112/89 Upjohn

11. Urteil des Europäischen Gerichtshofes vom 9. Juni 2005, verbundene Rechtssache C-211/03, C-316/03 und C-318/03, Randnr. 54, Tenor 4

12. Urteil des Oberverwaltungsgerichts NRW vom 17. März 2006-13 A 1977/02

13. Urteile des Bundesverwaltungsgerichtes (BVerwG) vom 25. Juli 2007 - 3 C 23.06, 3 C 22.06 und 3 C 21.06

14. Urteil des Bundesgerichtshofes (BGH) vom 11. Juli 2002-IZR34/01

15. Urteil des BVerwG vom 25. Juli 2007 - 3C 23.06, Randnr. 23

16. Schlussanträge des Generalanwalts Geelhoed vom 3. Februar 2005. HLH Warenvertriebs $\mathrm{GmbH}$ (C211/03) und Orthica BV (C-299/03 und C-316/03 bis (-318/03) gegen Bundesrepublik Deutschland. Ersuchen um Vorabentscheidung: Oberverwaltungsgericht NRW. Verbundene Rechtssachen C-211/03, C-299/03 und C-316/03 bis C-318/03

17. Urteil des BVerwG vom 25. Juli $2007-3$ C 22.06

18. Urteil des BVerwG vom 16. Mai 2007-3C 34.06

19. Urteil des Europäischen Gerichtshofes vom 15 November 2007-Rs C-319/05- „Knoblauchkapseln“

20. Urteil des Europäischen Gerichtshofes vom 15. Januar 2009-Rs C-140/07 „Red Rice“

21. Urteil des Verwaltungsgerichtes Köln vom 05. August 2014-7 K5469/12

22. Urteil des Oberverwaltungsgerichtes des Saarlandes vom 3. Februar 2006-3R7/05- "Weihrauch"

23. Urteil des Europäischen Gerichtshofes vom 30. April 2009-RsC-27/08- "Weihrauch"

24. Urteil des Europäischen Gerichtshofes vom 10. Juli 2014-Rs C-358/13 und C-181/14 - „legal highs"

25. Urteil des BVerwG vom 20. November 2014 - 3C 26.13- "elektrische Zigarette" 